\title{
Evolutionary games with coordination and self-dependent interactions
}

\author{
Balázs Király ${ }^{1}$ and György Szabó ${ }^{2}$ \\ ${ }^{1}$ Budapest University of Technology and Economics, Budafoki út 8, H-1111 Budapest, Hungary \\ ${ }^{2}$ Institute of Technical Physics and Materials Science, Centre for Energy Research, Hungarian Academy of Sciences, \\ P.O. Box 49, H-1525 Budapest, Hungary
}

(Received 10 August 2016; revised manuscript received 3 November 2016; published 4 January 2017)

\begin{abstract}
Multistrategy evolutionary games are studied on a square lattice when the pair interactions are composed of coordinations between strategy pairs and an additional term with self-dependent payoff. We describe a method for determining the strength of each elementary coordination component in $n$-strategy potential games. Using analytical and numerical methods, the presence and absence of Ising-type order-disorder phase transitions are studied when a single pair coordination is extended by some types of self-dependent elementary games. We also introduce noise-dependent three-strategy equivalents of the $n$-strategy elementary coordination games.
\end{abstract}

DOI: 10.1103/PhysRevE.95.012303

\section{INTRODUCTION}

In multiagent evolutionary games [1-4] the players are located on the sites of a lattice or graph, play two-player games with their nearest neighbors, and are allowed to change their strategies by following a dynamical rule based on their payoffs. It is already clarified that the resulting stationary state depends on the number of strategies, the payoff matrix, the dynamical rule, and the connectivity structures defining the interacting pairs. Until now the systematic analysis of the possible stationary states was limited to two-strategy systems because for $n$ strategies the number of payoff parameters increases with $n^{2}$. The concept of matrix decomposition [5-8], however, provides an efficient way to explore possible behaviors in the whole space of payoff parameters. In this approach the payoff matrix is built up as a linear combination of orthogonal basis matrices representing four types of interactions: coordination, self-dependence, cross dependence, and cyclic dominance. Within these types of interactions there are games that can be transformed into each other by relabeling the strategies. These inherent symmetries imply equivalent behavior in different regions of the parameters and significantly reduce the effort necessary to explore the possible system behaviors as a function of payoff parameters.

Such an anatomy of matrix interactions becomes fruitful if it provides deep insight into how evolutionary games behave, how their strategy distributions evolve, what spatial structures emerge during this evolution, whether they possess phase transitions, etc. The first steps of this systematic approach imply studying the general behavior of a macroscopic system if the pair interactions are described by the simplest basis games. It is known [8], for example, that multiagent evolutionary games (on a lattice or regular graph) can be exactly described by the behavior of a single player if the uniform pair interaction is defined as a linear combination of self- and cross-dependent components if the logit rule controls strategy updates [8-13]. Among potential games, realistic pair interactions are represented by the class of coordination games. The effects of increasing the number of strategies in elementary coordination games have already been investigated [14]. In the spirit of the above-mentioned exploration process, our analysis will now be focused on potential games composed of an elementary coordination-type game and additional games with self-dependent payoffs. Our recent research aims to clarify the interplay between these two elementary interactions. This bottom-up approach can shine a light on general relationships and universal features that also occur in well-investigated physical systems.

The coordination-type interaction is already present among symmetric two-strategy games and it represents systems that are equivalent to widely studied Ising-type models $[15,16]$ (which crop up in research fields ranging from magnetism to biology to sociophysics [17-24]) and exhibit similar thermodynamic behavior if the logit rule controls strategy updates [8-13]. In the original coordination game [25-29] the two players independently choose one of two strategies and they are paid 1 (unit of payoff) if they both have chosen the same option and lose 1 otherwise. Similar coordination-type interactions can be found among the $2 \times 2$ subgames of certain $n$-strategy games where the players are restricted to use only their $i$ th and $j$ th strategies $(1 \leqslant i<j \leqslant n)$. In the corresponding elementary coordination games all the other payoff components are zero. Evidently, elementary coordination games with another strategy pair (e.g., $i^{\prime}$ and $j^{\prime}$ ) exhibit the same behavior (noise dependence for the logit rule) on any graph. The analysis of these systems on a square lattice has revealed [14] that a continuous (Ising-type) order-disorder phase transition can be observed when the noise level is increased if $n$ is less than a threshold value $\left(n<n_{\text {th }}\right)$. Otherwise, this system undergoes a first-order phase transition at a critical noise level $K_{c}(n)$ that vanishes as $K_{c}(n) \simeq 2 / \ln n$ if $n \rightarrow \infty$.

It will be shown that the addition of a self-dependent component to elementary coordination games on a square lattice can change the order of, and even abolish, the orderdisorder phase transition. In Sec. II we give a detailed overview of the models studied. Section III introduces the methods we use to study these models and presents our results. Section IV establishes the equivalence between an $n$-strategy elementary coordination game and a three-strategy elementary coordination game combined with suitably chosen noise-level-dependent self-dependent components. Finally, in Sec. V we summarize our main conclusions.

\section{MODELS AND GENERAL FEATURES}

We consider spatial evolutionary games played by equivalent players who are located on the $N=L \times L$ sites of a square 
lattice with periodic boundary conditions $[2,4,8]$. Each player $x$ can choose one of $n$ pure strategies $\mathbf{s}_{x}$, which are usually denoted by the $n$-dimensional Cartesian unit vectors. In other words, the $i$ th strategy $(i=1,2, \ldots, n)$ will be denoted by the $i$ th unit vector. Using this formalism, the income of the player located on site $x$ is given by

$$
\tilde{u}_{x}\left(\mathbf{s}_{x}\right)=\sum_{\delta} \mathbf{s}_{x} \cdot \mathbf{A} \mathbf{s}_{x+\delta}
$$

where the summation runs over the nearest neighbors located at sites $x+\delta$ and the payoffs are defined by an $n \times n$ matrix A [2].

In the following we will consider combinations of two types of basis games. Namely, a coordination-type game with a unit strength is perturbed by one or two additional self-dependent components with adjustable strength. All these symmetric two-player games are potential games and their corresponding potential matrix $\mathbf{V}$ can be determined [8]. The total potential of the whole multiplayer system is given by

$$
U(\mathbf{s})=\frac{1}{2} \sum_{x, \delta} \mathbf{s}_{x} \cdot \mathbf{V} \mathbf{s}_{x+\delta},
$$

when the individual players' strategies are defined by the strategy profile $\mathbf{s}=\left\{\mathbf{s}_{x}\right\}$. This potential $U(\mathbf{s})$ plays the role of the negative potential energy in Ising-type models and it summarizes the individual incentives of the active players for consecutive unilateral strategy changes, namely, if player $x$ changes strategy from $\mathbf{s}_{x}$ to $\mathbf{s}_{x}^{\prime}$ then

$$
\tilde{u}_{x}\left(\mathbf{s}_{x}\right)-\tilde{u}_{x}\left(\mathbf{s}_{x}^{\prime}\right)=U(\mathbf{s})-U\left(\mathbf{s}^{\prime}\right),
$$

where $\mathbf{s}_{y}=\mathbf{s}_{y}^{\prime}$ for all $y \neq x$ in the strategy profiles $\mathbf{s}$ and $\mathbf{s}^{\prime}$.

In the present systems the players revise their strategies in random sequential order following the logit rule, according to which a randomly selected player $x$ changes strategy to $\mathbf{s}_{x}^{\prime}$ with a probability

$$
w\left(\mathbf{s}_{x}^{\prime}\right)=\frac{e^{\tilde{u}_{x}\left(\mathbf{s}_{x}^{\prime}\right) / K}}{\sum_{\mathbf{s}_{x}} e^{\tilde{u}_{x}\left(\mathbf{s}_{x}\right) / K}} .
$$

This update rule exponentially favors higher individual income under the assumption that the strategy profile remains unchanged in the player's neighborhood [12,30-33]. The parameter $K$ quantifies the uniform strength of noises (a player's perception and decision making can both be imperfect, i.e., noisy) present in the system. A system governed by this evolutionary rule evolves into the Boltzmann distribution $[9,34]$ in which the probability $p(\mathbf{s})$ of finding the system in strategy profile $\mathbf{s}$ is determined by the following expression:

$$
p(\mathbf{s})=\frac{e^{U(\mathbf{s}) / K}}{\sum_{\mathbf{s}^{\prime}} e^{U\left(\mathbf{s}^{\prime}\right) / K}} .
$$

All coordination-type interactions [6] can be composed of elementary coordination games that are defined by the payoff matrices $\mathbf{d}(p, q ; n)$ and express Ising-type coordination between the strategies $p$ and $q(p<q)$, namely,

$$
d_{i j}(p, q ; n)=\left\{\begin{aligned}
1 & \text { for } i=j=p \\
1 & \text { for } i=j=q \\
-1 & \text { for } i=p, j=q \\
-1 & \text { for } i=q, j=p \\
0 & \text { otherwise. }
\end{aligned}\right.
$$

A linear combination of these elementary components takes the form [35]

$$
\begin{aligned}
\mathbf{A}^{\text {(coord })} & =\sum_{1 \leqslant p<q \leqslant n} \alpha_{p q} \mathbf{d}(p, q ; n) \\
& =\left(\begin{array}{cccc}
\beta_{1} & -\alpha_{12} & \cdots & -\alpha_{1 n} \\
-\alpha_{12} & \beta_{2} & \cdots & -\alpha_{2 n} \\
\vdots & \vdots & \ddots & \vdots \\
-\alpha_{n 1} & -\alpha_{n 2} & \cdots & \beta_{n}
\end{array}\right)
\end{aligned}
$$

and defines a general coordination-type game. Notice that in this combination the strength of each elementary $\mathbf{d}(p, q ; n)$ component is directly given by the corresponding off-diagonal element of $\mathbf{A}^{\text {(coord) }}$, while the matrix's diagonal elements are $\beta_{p}=\sum_{q \neq p} \alpha_{p q}=\sum_{q \neq p} \alpha_{q p}$ because the sum of payoffs is zero in each row and column. It is worth noting that Eq. (7) gives us a method to determine the strength of each elementary coordination game in a payoff matrix $\mathbf{A}$ after separating the coordination-type interactions $[7,8]$.

The class of general coordination-type games also contains games that actually lead to anticoordinated spatial strategy distributions [7], e.g., an elementary coordination game with negative strength. In "real" coordination games the maximal value of $A_{i j}$ is located in the main diagonal of the payoff matrix [e.g., $\max \left(A_{i j}\right)=\beta_{1}$ ] and the corresponding strategy forms a homogeneous phase at low noise levels that varies smoothly towards the disordered phase if $K$ is increased. In general, the (Ising- or Potts-type) equivalence of two (or more) homogeneous states is eliminated by varying the values of $\alpha_{p q}$. On the other hand, if $\max \left(A_{i j}\right)=-\alpha_{p q}$ we get an anticoordination game in which the strategy pair $(p, q)$ can form two equivalent sublattice ordered strategy arrangements (at low noise levels on a square lattice), reminiscent of an antiferromagnetic spin system. Similar strategy distributions can also occur on other bipartite lattices or graphs as well. Such antiferromagnetic systems are in fact equivalent to ferromagnetic ones, as exchanging the labels $p$ and $q$ on one of the sublattices transforms anticoordination into coordination and vice versa [15]. The anticoordinated systems always possess Ising-type critical phase transitions because the twofold degenerated ground state is preserved by the application of a homogeneous magnetic field (or self-dependent components detailed below) if its strength does not exceed a threshold value [36-39]. Similar behavior is expected for $n>2$.

Self-dependent game components can in general represent subsidies or taxes, depending on their effect on player income. The linear combinations of these types of interactions are represented by matrices $\mathbf{A}^{\text {(self) }}$ composed of rows with homogeneous payoffs, that is, $A_{i j}^{\text {(self) }}=h_{i}$. The corresponding elementary game components are described by the following payoff matrices:

$$
e_{i j}(p ; n)= \begin{cases}1 & \text { for } i=p \\ 0 & \text { otherwise. }\end{cases}
$$

In this formalism the self-dependent games can be defined as

$$
\mathbf{A}^{(\text {self })}=\sum_{p=1}^{n} h_{p} \mathbf{e}(p ; n) .
$$


It is noteworthy that the all 1 component belongs to this subset of games and represents an irrelevant term [8]. Here the roles of $h_{j}$ resemble the external fields in Potts models [40]. It is known that the potential matrix of self-dependent games is $\mathbf{V}^{\text {(self) }}=\mathbf{A}^{\text {(self) }}+\mathbf{A}^{\text {(self) } T}$ (the index $T$ refers to transposition) [8].

The solution of a spatial evolutionary game made up only of self-dependent components $\mathbf{A}=\mathbf{A}^{\text {(self) }}$ can be reduced to a one-site problem because the players' payoffs are solely defined by their own strategy choices. For example, in the logit-rule-driven evolutionary game defined by $\mathbf{A}=h_{1} \mathbf{e}(1 ; n)$ the equilibrium strategy frequencies on a square lattice are given by Eq. (5) as

$$
\begin{aligned}
& \rho_{1}=\frac{e^{4 h_{1} / K}}{e^{4 h_{1} / K}+(n-1)}, \\
& \rho_{p}=\frac{1}{e^{4 h_{1} / K}+(n-1)} \quad \text { for } p>1 .
\end{aligned}
$$

The prefactor 4 in the exponent corresponds to the number of nearest neighbors in the square lattice.

Now we study the effect of adding self-dependent game components to the aforementioned coordination-type interaction defined by Eq. (6) while preserving the equivalence of the $n-2$ neutral strategies. More quantitatively, we study evolutionary games defined by the payoff matrices

$$
\begin{gathered}
\mathbf{A}^{\prime}=\mathbf{d}(1,2 ; n)+h^{\prime} \mathbf{e}(1 ; n), \\
\mathbf{A}^{\prime \prime}=\mathbf{d}(1,2 ; n)+h^{\prime \prime}[\mathbf{e}(1 ; n)+\mathbf{e}(2 ; n)] .
\end{gathered}
$$

In the previous notation the interaction of $\mathbf{A}^{\prime}$ includes a selfdependent component with $h_{1}=h^{\prime}$ and $h_{p}=0$ if $p>1$ and with $\mathbf{A}^{\prime \prime} h_{1}=h_{2}=h^{\prime \prime}$ and $h_{p}=0$ if $p>2$. Evidently, the system with $h_{1}=0, h_{2}=h^{\prime}$, and $h_{p}=0$ for $p>2$ can be mapped onto $\mathbf{A}^{\prime}$ by exchanging the strategy labels 1 and 2 .

The game of $\mathbf{A}^{\prime \prime}$ has two equivalent preferred Nash equilibria as both the coordination and self-dependent components favor strategy 1 or 2 equally. In lattice systems this interaction results in a homogeneous state of strategy 1 (or 2) in the zero noise limit and an order-disorder phase transition is expected when $K$ is increased. This Ising-type critical phase transition is generally quantified by the variation of an order parameter $m=\rho_{1}-\rho_{2}$, where $\rho_{i}$ measures the frequency of strategy $i$ in the whole system. For $h^{\prime \prime}=0$ the previous analyses [14] have indicated that $m=0$ above the critical noise level $\left(K>K_{c}\right)$, while $\rho_{i}=\rho_{j}$ if $2<i<j \leqslant n$ and all $\rho_{i}$ go to $1 / n$ if $K \rightarrow \infty$. Due to the identical symmetries similar behavior is expected at a critical noise level dependent on the value of $h^{\prime \prime} \neq 0$. Notice, furthermore, that the effect of this interaction $\mathbf{A}^{\prime \prime}$ is equivalent to the one where the self-dependent components are increased by a constant value $\left(A_{i j}^{\text {(self) }} \rightarrow A_{i j}^{\text {(self) }}+c\right)$ that may even be chosen as $c=-h^{\prime \prime}$. The latter model has a self-dependent component supporting or suppressing the neutral strategies equally.

If the interaction is given by $\mathbf{A}^{\prime}$ then the self-dependent component favors strategy 1 (2) if $h^{\prime}>0\left(h^{\prime}<0\right)$. This type of self-dependent interaction smooths out the critical phase transition in a way resembling the effect of an external magnetic field in the two-dimensional Ising model.

\section{METHODS AND RESULTS}

Cluster variation methods (for details see [8,41-43]) determine the equilibrium configuration probabilities (5) by finding the extremal values of a thermodynamic potential, in our case

$$
\Phi=\mathcal{U}+K \mathcal{S}
$$

where $\mathcal{U}$ and $\mathcal{S}$ are the average value of the potential $\left[\mathcal{U}=\sum_{\mathbf{s}} p(\mathbf{s}) U(\mathbf{s})\right]$ and entropy $\left[\mathcal{S}=-\sum_{\mathbf{s}} p(\mathbf{s}) \ln p(\mathbf{s})\right]$, respectively. The mean-field approximation (MFA) and pair approximation (PA) methods use one-site probabilities $p_{1}(i)=$ $\rho_{i}$ of finding strategy $i$ at any site (on the translation invariant lattice) and two-site configuration probabilities $p_{2}(i, j)$ of finding the strategy pair $(i, j)$ on two neighboring sites to approximate the aforementioned thermodynamic potential.

The numerical solutions of the MFAs and PAs are presented in Fig. 1 for several parameters if the interactions are defined by the payoff matrix $\mathbf{A}^{\prime \prime}$. In general, these results show
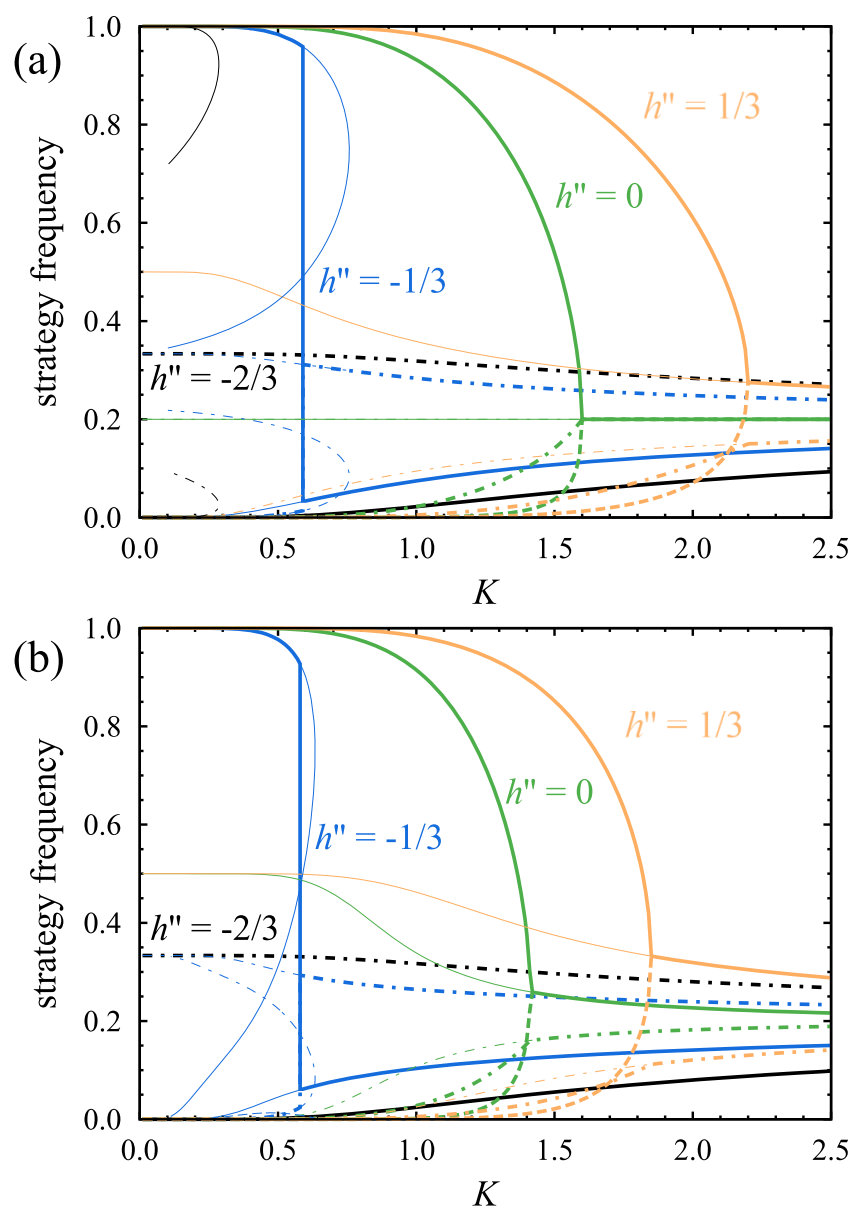

FIG. 1. Strategy frequencies as a function of $K$ for $n=5$ and $h^{\prime \prime}=-2 / 3,-1 / 3,0$, and $1 / 3$ [plotted with black, blue (dark gray), green (medium gray), and orange (light gray) lines, respectively] in the model defined by the payoff matrix (12). The lines denote solutions of the (a) MFA and (b) PA, with thick and thin lines corresponding to stable and unstable states respectively. Solid and dashed lines belong to the two coordinated strategies and dash-dotted lines represent neutral strategies. The vertical lines indicate first-order phase transitions. The unstable solution of the PA at $h^{\prime \prime}=-2 / 3$ is covered by the other plots. 
qualitatively similar predictions for the equilibrium properties of the system and the MFA predicts higher critical transition points in comparison with those found by PAs. We have to emphasize that both the MFA and PA predict a disordered state of the neutral strategies, with $\rho_{1}=\rho_{2}=0$ and $\rho_{3}=$ $\rho_{4}=\rho_{5}=1 / 3$, in the low noise limit for low enough values of $h^{\prime \prime}$, namely, if $h^{\prime \prime}<0$. Notice, furthermore, that the unstable solutions of the MFA reserve the symmetry $\rho_{1}=\rho_{2}$, which eliminates the contributions of $\mathbf{d}(1,2 ; n)$. Thus, these unstable solutions are only determined by the value of $h^{\prime \prime}$.

This system exhibits three different types of behavior as $K$ is increased, depending on the strength $h^{\prime \prime}$ of the self-dependent component. If $h^{\prime \prime}>h_{c}^{\prime \prime}$ then the system undergoes a continuous order-disorder phase transition, which becomes a first-order one if $h_{c}^{\prime \prime}>h^{\prime \prime}>h_{f}^{\prime \prime}$ and disappears in the $h_{f}^{\prime \prime}>h^{\prime \prime}$ case. The critical noise level $K_{c}$ of the phase transition decreases as $h^{\prime \prime}$ decreases.

In the low-noise limit in one of the ordered structures $\rho_{1} \rightarrow$ 1 and $\rho_{2}<\rho_{i}=\rho_{3}$ for $2<i \leqslant n$, while in the disordered phase (if $K>K_{c}$ or $h^{\prime \prime}<h_{f}^{\prime \prime}$ ) $\rho_{1}=\rho_{2}<\rho_{i}=\rho_{3}$, at least in the high noise limit $(K \rightarrow \infty)$. Notice that a strong enough negative $h^{\prime \prime}$ field and high entropy can stabilize the disordered phase even at low noise levels. A similar mechanism can be utilized for high-entropy alloys [44-46], which are considered a promising family of materials for several technical purposes.

One can easily determine the value of $h_{f}^{\prime \prime}$ by comparing the average values of the potential $\mathcal{U}$ the system has in the predicted solutions if $K \rightarrow 0$. In one of the ordered states of the coordinated strategies (e.g., $\rho_{1}=1$ and $\rho_{i}=$ 0 for $i>1) \mathcal{U} / N=2+4 h^{\prime \prime}$. On the other hand, in the disordered state of the neutral strategies $\mathcal{U} / N=0$. Comparing the average potential of the two competing ground states yields that both of the ordered (homogeneous) states of the coordinated strategies are equivalent stable ground states if $h^{\prime \prime}>h_{f}^{\prime \prime}=-1 / 2$ (independently of the value of $n$ ); otherwise the disordered arrangement of neutral strategies becomes the ground state.

We have yet to find a similar analytic expression that describes the strategy number dependence of $h_{c}^{\prime \prime}$. At the same time the numerical evaluation of this dependence would require extensive numerical calculations even in the MFA and PA. Obtaining exact results is even more time consuming due to the critical slowing down of Monte Carlo simulations near the transition point. Determining $h_{c}^{\prime \prime}$ as a function of the strategy number $n$ goes beyond the scope of the present paper.

As mentioned above, in the MFA the high-entropy stationary states of the system of $\mathbf{A}^{\prime \prime}$ depend only on the self-dependent component and the strategy frequencies can be given as

$$
\begin{aligned}
& \rho_{1}=\rho_{2}=\frac{e^{4 h^{\prime \prime} / K}}{2 e^{4 h^{\prime \prime} / K}+(n-2)}, \\
& \rho_{i}=\frac{1}{2 e^{4 h^{\prime \prime} / K}+(n-2)} \quad \text { for } i>2 .
\end{aligned}
$$

Notice that the sign of $h^{\prime \prime}$ determines the numerical order of these strategy frequencies and consequently their $K \rightarrow 0$ limits: If $h^{\prime \prime}$ is positive $\rho_{1} \rightarrow 1 / 2$ and $\rho_{i} \rightarrow 0$, whereas for negative values of $h^{\prime \prime}$, as neutral strategies become best response options against neutral strategies, we find that $\rho_{1} \rightarrow 0$ and $\rho_{i} \rightarrow 1 /(n-2)$.

By considering the Taylor series expansion of the thermodynamic potential around these solutions we can determine the critical temperature of continuous phase transitions. Thus one can derive an implicit equation for $K_{c}$ in the framework of the MFA that takes the following form:

$$
K_{c}^{(\mathrm{MF})}=8 \rho_{1}\left(K_{c}^{(\mathrm{MF})}\right)=\frac{8 e^{4 h^{\prime \prime} / K_{c}^{(\mathrm{MF})}}}{2 e^{4 h^{\prime \prime} / K_{c}^{(\mathrm{MF})}}+(n-2)} .
$$

In agreement with the traditional predictions of the MFAs, the Taylor series expansion can also be used to justify that the $K$ dependence of the order parameter follows the well-known behavior, i.e., $\left|\rho_{1}-\rho_{2}\right| \propto\left(K_{c}^{(\mathrm{MFA})}-K\right)^{1 / 2}$ if the transition is continuous and $K \rightarrow K_{c}^{\text {(MFA) }}$ from below.

The introduction of the symmetry-breaking self-dependent component [given by $\mathbf{A}^{\prime}$ in Eq. (11)] can also lead to the disappearance of the critical phase transition present in evolutionary spatial elementary coordination games. In fact, a phase transition can only remain present in such systems if the self-dependent component is not too beneficial $\left(h^{\prime}<h_{c}^{\prime}\right)$. According to previous Monte Carlo simulations [14] for $h^{\prime}=$ 0 , the present system exhibits a first-order phase transition if the number of strategies $n$ is above a threshold value $n_{\text {th }}=27$. In this case the first-order phase transition can be observed for $h^{\prime}<h_{c}^{\prime} \geqslant 0$. The MFA and PA, however, predict lower threshold values in the number of strategies, quantitatively, $n_{\mathrm{th}}^{(\mathrm{MFA})}=6$ and $n_{\mathrm{th}}^{(\mathrm{PA})}=10$.

In Fig. 2 the results of the MFA and PA illustrate clearly how the critical phase transition is smoothed out in the presence of $h^{\prime}$ if the strategy number is below its threshold value. The unperturbed elementary coordination game $\left(h^{\prime}=0\right)$ has two equivalent ordered strategy arrangements if $K \rightarrow 0$.

As mentioned above, this equivalence is broken if we introduce an $h^{\prime} \neq 0$ field. The $h^{\prime}>0$ case favors strategy 1 . Consequently, $\rho_{1}$ is the highest among the strategy frequencies in the stable state and it decreases monotonically and continuously from 1 to $1 / n$ as $K$ is increased (see the dash-dotted lines for both approximations). Also, $\rho_{1}>\rho_{i}=\rho_{3}>\rho_{2}(i>3)$ at all noise levels, which is different from the $h^{\prime}=0$ game, where strategy 2's frequency is equal to strategy 1's, both of which exceed the equal frequencies of the neutral strategies. At the PA level we have also found unstable states that only exist at low enough noise levels and also exhibit some unexpected behavior. In these states strategy 2 has the highest frequency and $\rho_{2}$ goes to 1 as $K$ is decreased to 0 but it is not a monotonic function of the noise level: $\rho_{2}$ briefly increases close to the right edge of its domain. The thin orange (light gray) lines of Fig. 2 correspond to the strategy frequencies of one such unstable state.

An $h^{\prime}<0$ system has very similar properties (for an example see the dashed lines in Fig. 2), with a few differences. In this case the choice of strategy 1 is penalized, which essentially reverses the roles of the two coordinated strategies in comparison with positive $h^{\prime}$ games. This means that $\rho_{2}>$ $\rho_{i}=\rho_{3}>\rho_{1}$ (again $i>3$ ), the phase transition is smoothed out, strategy 2's frequency monotonically decreases to $1 / n$ in the $K \rightarrow \infty$ limit, and even the unstable states in the PA are similar. However, the frequency of the neutral strategies 

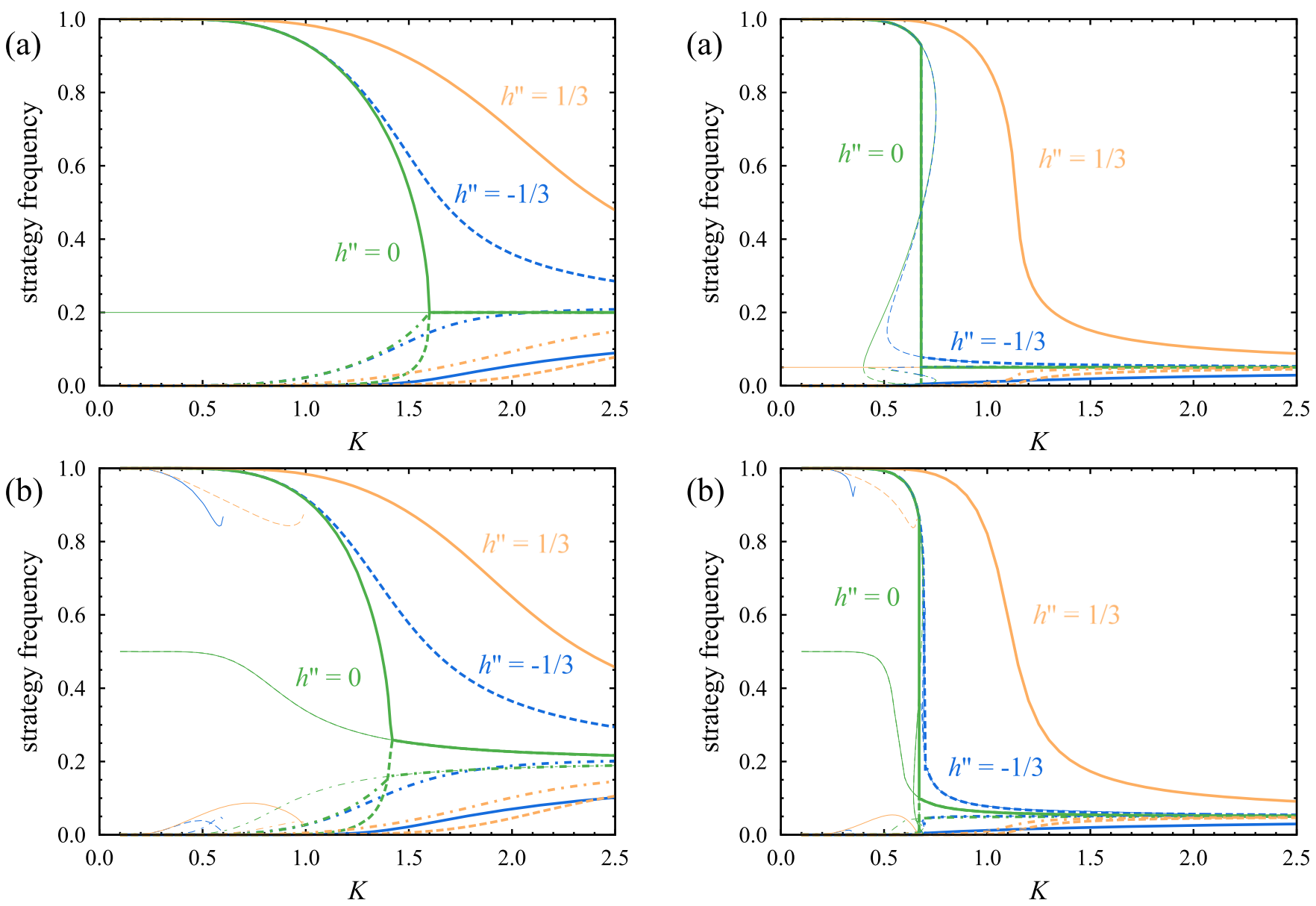

FIG. 2. Predictions of the (a) MFA and (b) PA for the strategy frequencies vs $K$ for $n=5$ and $h^{\prime}=-1 / 3$ [blue (dark gray) lines], $h^{\prime}=0$ [green (medium gray) lines], and $h^{\prime}=1 / 3$ [orange (light gray) lines] if the payoff matrix is defined by Eq. (11). Dash-dotted lines correspond to neutral strategies and solid and dashed lines represent the frequencies of strategies 1 and 2, respectively. Thick and thin lines denote stable and unstable states, respectively.

is not a monotonic function of the noise level anymore: At a finite value of $K$ it rises above its $K \rightarrow \infty$ limit of $1 / n$, which implies the existence of an inflection.

In contrast to the examples above, the first-order phase transition can still be observed as it is demonstrated in Fig. 3 for $n=20$. For such a high value of $n$ the MFA and PA both predict a first-order phase transition for $h^{\prime}=0$ [see the solid lines in Figs. 3(a) and 3(b)] and these transitions remain present for all $h^{\prime}$ below a positive $h_{c}^{\prime}$. The preferred coordinated strategy forms a homogeneous state in the low noise limit. As $K$ is increased this strategy's frequency starts to decrease and at $K_{\mathrm{c}}$ it abruptly drops and then goes to $1 / n$ as $K$ is further increased, as the dashed lines show in Fig. 3. Our numerical data indicate that the value of $h^{\prime}$ (as long as it remains below its critical $h_{\mathrm{c}}^{\prime}$ strength) only very slightly affects the critical noise level of the first-order transition. Nonzero fields also change the character of the transition. If $h^{\prime}=0$ the transition occurs due to the competition of two distinct states, a low-entropy and a high-entropy one. On the other hand, for finite $h^{\prime}$ the low- and high-temperature stable states are described by two branches

FIG. 3. Strategy frequencies as a function of $K$ for $n=20$ and $h^{\prime}=-1 / 3,0$, and $1 / 3$ [plotted with blue (dark gray), green (medium gray), and orange (light gray) lines, respectively] in the model defined by Eq. (11). Solid (strategy 1) and dashed (strategy 2) lines belong to the two coordinated strategies and the dash-dotted lines represent neutral strategies. The lines correspond to the (a) MFA and (b) PA solutions, with thick and thin lines denoting stable and unstable states, respectively, as in Figs. 1 and 2. The vertical lines indicate first-order phase transitions.

of continuous multivariable strategy frequency functions. An example is plotted with dashed lines in Fig. 3. Above $K_{\mathrm{c}}$ the higher-entropy branch's thermodynamic potential becomes the highest and this is reflected in the presence of the first-order transition. In the presence of a strong enough positive $h^{\prime}$ field the phase transition is still abolished and the system behaves like the lower- $n$ positive- $h^{\prime}$ games discussed previously.

\section{THREE-STRATEGY EQUIVALENTS OF ELEMENTARY COORDINATION GAMES}

The observations of Sec. III clearly suggest a similarity between the increase of $n$ for the absence of self-dependent components and the variation of the strengths of selfdependent components for $n=3$. For both cases the system is well characterized by the strategy frequencies $\rho_{1}$ and $\rho_{2}$ because the third or all the other neutral strategy frequencies are defined by the conditions $\sum_{i=1}^{n} \rho_{i}=1$ and $\rho_{i}=\rho_{3}$ if 
$i \geqslant 3$. Indeed, we can find a direct connection between these two sets of games, at least at the level of the MFA.

Let us first consider the spatial game defined by $\mathbf{A}=$ $\mathbf{d}(1,2 ; n)$. In the MFA the specific thermodynamic potential of this system can be written as

$$
\begin{aligned}
\frac{1}{N} \Phi^{(\mathrm{MF})}(n)= & 2\left(\rho_{1}-\rho_{2}\right)^{2} \\
& -K\left[\rho_{1} \ln \rho_{1}+\rho_{2} \ln \rho_{2}\right. \\
& \left.+\left(1-\rho_{1}-\rho_{2}\right) \ln \frac{1-\rho_{1}-\rho_{2}}{n-2}\right] .
\end{aligned}
$$

Now we turn our attention to the game defined by Eq. (12) for $n=3$. In this case the above formula for $n=3$ is extended by the contribution of the average potential of the self-dependent term, thus

$$
\begin{aligned}
\frac{1}{N} \Phi^{\prime \prime(\mathrm{MF})}(3)= & 2\left(\rho_{1}-\rho_{2}\right)^{2}+4 h^{\prime \prime}\left(\rho_{1}+\rho_{2}\right) \\
& -K\left[\rho_{1} \ln \rho_{1}+\rho_{2} \ln \rho_{2}\right. \\
& \left.+\left(1-\rho_{1}-\rho_{2}\right) \ln \left(1-\rho_{1}-\rho_{2}\right)\right] .
\end{aligned}
$$

Disregarding a constant, the $\rho_{1}$ and $\rho_{2}$ dependences of $\Phi^{(\mathrm{MF})}(n)$ and $\Phi^{\prime \prime(\mathrm{MF})}(3)$ coincide if

$$
h_{n}^{\prime \prime}=-\frac{\ln (n-2)}{4} K \text {. }
$$

One way to interpret this result is that virtual neutral strategies can be simulated in a three-strategy (two coordinated strategies and one neutral strategy) system by introducing a noise-level-dependent self-dependent component of strength $h_{n}^{\prime \prime}$ that retains the symmetries. One can also think of this noise-level-dependent game component as a substitute for the excess entropy arising from the distinctiveness of $n-2$ neutral strategies.

On the square lattice the above MFAs predict an equivalent $K$ dependence for the games with payoffs $\mathbf{A}=\mathbf{d}(1,2 ; n)$ and $\mathbf{A}^{\prime \prime}$ defined by (12) for $n=3$ and

$$
\mathbf{A}^{\prime \prime \prime}=\mathbf{d}(1,2 ; 3)-h_{n}^{\prime \prime} \mathbf{e}(3,3)
$$

if the $K$-dependent $h_{n}^{\prime \prime}$ is given by Eq. (18). Notice that the second and third systems can be mapped onto each other by adding a suitable constant to each matrix component.

Surprisingly, the predicted equivalence remains valid on the square lattice as indicated by Monte Carlo simulations for $n=6$ in Fig. 4. For the plotted data the statistical errors are smaller than the symbol size, except the close vicinity of the transition point.

The quantitative analysis of all the possible transition probabilities given by Eq. (4) has justified that one can merge the $n-2$ interchangeable neutral strategies into a single neutral state by introducing an effective three-strategy game that has the same potential. The detailed discussion of this calculation goes beyond the scope of the present work because the evolution into a given Boltzmann distribution is not affected by adding arbitrary cross-dependent components to the pair interactions. Furthermore, the uniform logit rule (4) can also be individualized in different ways, preserving the detailed balance in the Boltzmann distribution [8].

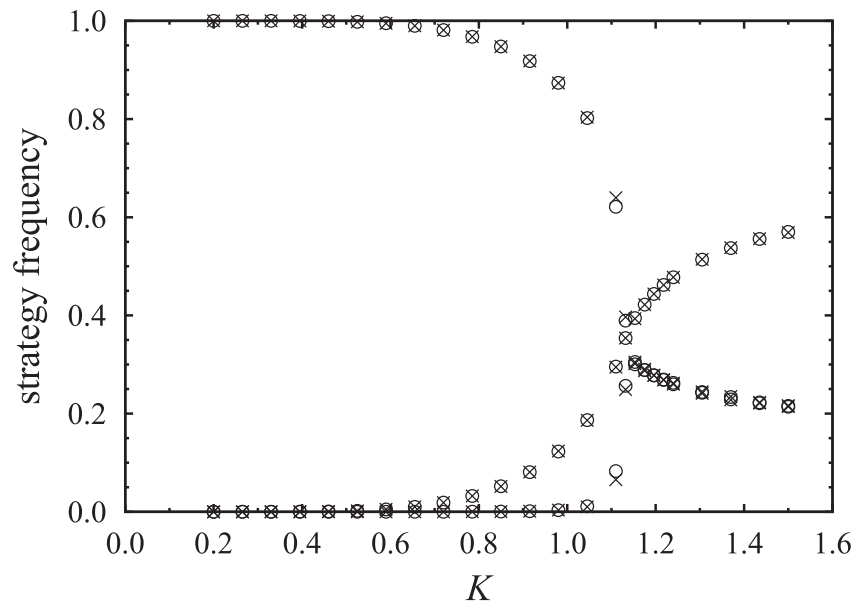

FIG. 4. Monte Carlo simulations on the square lattice show an equivalent $K$ dependence in the strategy frequencies when comparing the six-strategy systems with a pair interaction $\mathbf{d}(1,2 ; 6)$ (crosses) and its three-strategy equivalent (circles) given by Eq. (19). The frequencies of the four neutral strategies (for $n=6$ ) are merged into one to illustrate the agreement.

\section{SUMMARY}

In this paper we discussed a special subset of general $n$-strategy evolutionary potential games governed by the logit rule on a square lattice. These games are linear combinations of elementary coordination games (defining a coordinated strategy pair and neutral strategies) and certain self-dependent games. When summarizing the general features of the set of coordination games we found a method for determining the strength of each elementary coordination component that is not orthogonal to all the others. This simple method exploits the fact that the corresponding elementary games are described by sparse matrices.

Most of our results were obtained by using the MFA and PA methods. Cursory Monte Carlo simulations indicate that these methods give qualitatively correct predictions of the macroscopic behavior and properties of the games in question.

Elementary coordination games exhibit either a first-order or a continuous phase transition depending on the number of strategies. Our paper focuses on how this feature is modified by the addition of self-dependent game components that preserve the interchangeability of neutral strategies.

Adding a self-dependent component that retains the symmetry of neutral strategies but breaks the symmetry of the active strategies can have two consequences. First-order phase transitions, which are present if the number of strategies exceeds a threshold value, are preserved if the external field is not too strong. Otherwise the phase transitions are smoothed out. In both cases the preferred coordinated strategy forms a homogeneous ordered state in the zero noise limit.

The introduction of the other (symmetry-retaining and self-dependent) component has more possible outcomes. The strength of this component determines the order, and possibly even the presence, of the phase transition in these systems. If the external field's strength $h^{\prime \prime}$ is below $h_{f}^{\prime \prime}=-1 / 2$ the system's disordered high-entropy state becomes stabilized at all noise levels and no phase transitions are observed. In the $h_{f}^{\prime \prime}<h^{\prime \prime}<h_{c}^{\prime \prime}$ range a first-order phase transition 
appears that separates a low-entropy ordered state and a high-entropy disordered state. For $h_{c}^{\prime \prime}<h^{\prime \prime}$ this transition becomes continuous. The critical noise level of the transition increases with the field strength.

The three-strategy version of these games gives an opportunity to virtually simulate $n$-strategy elementary coordination games. By introducing an appropriate noise-level-dependent external field affecting the third strategy we can substitute the excess entropy of the additional neutral strategies of an $n$-strategy elementary coordination game. This effectively bunches $n-2$ equivalent but distinguishable strategies into one anomalous strategy with noise-level-dependent payoffs.

\section{ACKNOWLEDGMENT}

This work was supported by the Hungarian National Research Fund (Grant No. OTKA TK-120785).
[1] M. A. Nowak, Evolutionary Dynamics (Harvard University Press, Cambridge, 2006).

[2] G. Szabó and G. Fáth, Phys. Rep. 446, 97 (2007).

[3] C. P. Roca, J. A. Cuesta, and A. Sánchez, Phys. Life Rev. 6, 208 (2009).

[4] B. Allen and M. A. Nowak, EMS Surv. Math. Sci. 1, 113 (2014).

[5] O. Candogan, I. Menache, A. Ozdaglar, and P. A. Parrilo, Math. Oper. Res. 36, 474 (2011).

[6] G. Szabó, K. S. Bodó, B. Allen, and M. A. Nowak, Phys. Rev. E 90, 042811 (2014).

[7] G. Szabó, K. S. Bodó, B. Allen, and M. A. Nowak, Phys. Rev. E 92, 022820 (2015).

[8] G. Szabó and I. Borsos, Phys. Rep. 624, 1 (2016).

[9] L. E. Blume, Games Econ. Behav. 5, 387 (1993).

[10] L. E. Blume, Games Econ. Behav. 11, 111 (1995).

[11] D. Monderer and L. S. Shapley, Games Econ. Behav. 14, 124 (1996).

[12] J. S. Cramer, Logit Models From Economics and Other Fields (Cambridge University Press, Cambridge, 2003).

[13] D. Cheng, Automatica 50, 1793 (2014).

[14] G. Szabó and B. Király, Phys. Rev. E 93, 052108 (2016).

[15] C. Domb, in Phase Transitions and Critical Phenomena, edited by C. Domb and M. S. Green (Academic, London, 1974), Vol. 3, pp. 357-484.

[16] R. J. Glauber, J. Math. Phys. 4, 294 (1963).

[17] S. Galam, Y. Gefen, and Y. Shapir, Math. J. Sociol. 9, 1 (1982).

[18] A. V. M. Herz, J. Theor. Biol. 169, 65 (1994).

[19] W. A. Brock and S. N. Durlauf, Rev. Econ. Stud. 68, 235 (2001).

[20] G. Weisbuch and D. Stauffer, Physica A 384, 542 (2007).

[21] S. Galam and B. Walliser, Physica A 389, 481 (2010).

[22] S. Grauwin, D. Hunt, E. Bertin, and P. Jensen, Adv. Complex Syst. 14, 529 (2011).

[23] S. M. Krause and S. Bornholdt, Physica A 392, 4048 (2013).

[24] J.-P. Bouchaud, J. Stat. Phys. 151, 567 (2013).
[25] R. Gibbons, Game Theory for Applied Economists (Princeton University Press, Princeton, 1992).

[26] M. J. Osborn and A. Rubinstein, A Course in Game Theory (MIT Press, Cambridge, 1994).

[27] J. W. Weibull, Evolutionary Game Theory (MIT Press, Cambridge, 1995).

[28] R. Cooper, Coordination Games (Cambridge University Press, Cambridge, 1998).

[29] K. Sigmund, The Calculus of Selfishness (Princeton University Press, Princeton, 2010).

[30] G. W. Brown, in Activity Analysis of Production and Allocation, edited by T. C. Koopmans (Wiley, New York, 1951), pp. 373376.

[31] D. Monderer and L. S. Shapley, J. Econ. Theory 68, 258 (1996).

[32] W. H. Sandholm, Games 1, 3 (2010).

[33] G. Szabó and A. Szolnoki, J. Theor. Biol. 299, 81 (2012).

[34] L. E. Blume, Games Econ. Behav. 44, 251 (2003).

[35] S.-H. Hwang and L. Rey-Bellet, arXiv:1106.3552v2.

[36] E. Müller-Hartmann and J. Zittartz, Z. Phys. B 27, 261 (1977).

[37] X. N. Wu and F. Y. Wu, Phys. Lett. A 144, 123 (1990).

[38] J. L. Monroe, Phys. Rev. E 64, 016126 (2001).

[39] B. J. Lourenço and R. Dickman, J. Stat. Mech. (2016) 033107.

[40] F. Y. Wu, Rev. Mod. Phys. 54, 235 (1982).

[41] R. Kikuchi and S. Brush, J. Chem. Phys. 47, 195 (1967).

[42] T. Morita, Prog. Theor. Phys. Suppl. 115, 27 (1994).

[43] D. de Fontaine, in Theory and Applications of the Cluster Variation and Path Probability Methods, edited by J. L. Morin-Lopez and J. M. Sanchez (Springer, New York, 1996), pp. 125-144.

[44] R. Carroll, C. Li, C.-W. Tsai, J. Yeh, J. Antonaglis, B. A. W. Brinkman, M. LeBlanc, X. Xie, S. Chen, P. K. Liaw, and K. A. Dahmen, Sci. Rep. 5, 16997 (2015).

[45] O. N. Senkov, G. B. Wilks, D. B. Miracle, C. P. Chuang, and P. K. Liaw, Intermetallics 18, 1758 (2010).

[46] J.-W. Yeh, J. Met. 65, 1759 (2013). 